\section{Matrix-Isolierung und Eigenschaften des Kohlenmonosulfids}

\author{
R. Steudel
}

Institut für Anorganische und Analytische Chemie der Technischen Universität Berlin

(Z. Naturforschg. 21 b, 1106 [1966] ; eingegangen am 28. Juli 1966)

Für den Zerfall des Kohlenmonosulfids wurde bisher eine einfache Polymerisation zu (CS $)_{n}$ angenommen. Diese Vorstellung trifft nicht zu. Wir konnten das in einer HF-Entladung in $\mathrm{CS}_{2}$-Dampf $(60 \mathrm{kV}, 430 \mathrm{kHz})$ dargestellte CS durch Abschrecken auf $-190{ }^{\circ} \mathrm{C}$ in $\mathrm{CS}_{2}$-Matrix isolieren und durch sein IR-Spektrum nachweisen. Die Valenzschwingung liegt bei $v_{1}=1274 \mathrm{~cm}^{-1}$ Kraftkonstante $8,5 \mathrm{mdyn} / \AA \AA$, Bindungsgrad 2,2). Daraus folgt die Struktur

$$
|\mathrm{C}=\underline{\overline{\mathrm{S}}} \longleftrightarrow| \stackrel{\ominus}{\mathrm{C}} \equiv \stackrel{\oplus}{\mathrm{S}} \mid
$$

Die Absorptionen der Matrix liegen bei 1457 (m), 1509 (vs, $\left.v_{3}\right), 2103$ (vw), $2146\left(\mathrm{w}, v_{1}+v_{3}\right), 2290$ (vw, $\left.2 v_{2}+v_{3}\right) \mathrm{cm}^{-1}$. CS ist bei $-190^{\circ}$ in $\mathrm{CS}_{2}$ mehrere Stdn. beobachtbar, die Absorptionsintensität nimmt jedoch langsam ab $(0,5 \% / \mathrm{min})$. Bei $-165^{\circ}$ zerfällt CS in wenigen Sek. quantitativ. Die Zerfallsprodukte sind ein schwarzes $\mathrm{C}-\mathrm{S}$-Polymerisat und $\mathrm{C}_{3} \mathrm{~S}_{2}$, das durch seine Absorptionen bei 2058 und $1017 \mathrm{~cm}^{-1}$ nachgewiesen wurde ${ }^{1}$. Bei $-80^{\circ}$ läßt sich das $\mathrm{CS}_{2}$ weitgehend $\mathrm{ab}$ pumpen; beim Temperieren auf $20^{\circ}$ polymerisiert auch die Hauptmenge des $\mathrm{C}_{3} \mathrm{~S}_{2}$, und nur ein sehr kleiner Teil destilliert ab. Es liegt dann ein Gemisch niederer, polymerer Kohlenstoffsulfide vor. Dessen IR-Banden bei $460,810,1069,1420$ und $1660 \mathrm{~cm}^{-1}$ zeigen $(\mathrm{S}-\mathrm{S})-,(\mathrm{C}-\mathrm{S})-,(\mathrm{C}=\mathrm{S})$ - und $(\mathrm{C}=\mathrm{C})$-Bindungen an. Da sich Schwefel weder extrahieren noch bei $100{ }^{\circ} \mathrm{C}$ / $10^{-5}$ Torr heraussublimieren läßt, ist eine Kopolymerisation wahrscheinlich ${ }^{3}$. Der folgende Reaktionsmechanismus steht im Einklang mit thermodynamischen Überlegungen:

$$
\begin{aligned}
2 \mathrm{CS} & \longrightarrow \mathrm{C}_{2} \mathrm{~S}+\mathrm{S}_{1} \\
\mathrm{C}_{2} \mathrm{~S}+\mathrm{CS} & \rightarrow \mathrm{C}_{3} \mathrm{~S}_{2} \\
n \mathrm{~S}_{1} & \longrightarrow \mathrm{S}_{\mathrm{n}} \\
\mathrm{C}_{2} \mathrm{~S}+\mathrm{S}_{\mathrm{n}} & \longrightarrow \mathrm{C}_{\mathrm{X}} \mathrm{S}_{\mathrm{y}}
\end{aligned}
$$

CS zerfällt auch in der Gasphase (wahrscheinlich an den Gefäßwänden) rasch nach dem angegebenen Me- chanismus. $\mathrm{C}_{3} \mathrm{~S}_{2}$ wurde in dem aus der Entladung strömenden $\mathrm{Gas}$ massenspektroskopisch nachgewiesen. Der braune Wandbelag von $\mathrm{C}_{\mathrm{x}} \mathrm{S}_{\mathrm{y}}$ absorbiert, in $\mathrm{H}_{2} \mathrm{SO}_{4}$ gelöst, erwartungsgemäß bei $375-390 \mathrm{~nm}^{4}$. Da man somit auch unmittelbar hinter der Entladung ein Gemisch von $\mathrm{CS}, \mathrm{CS}_{2}$ und $\mathrm{C}_{3} \mathrm{~S}_{2}$ kondensiert, ist $x>y$. Das kohleartige $\mathrm{C}_{\mathrm{x}} \mathrm{S}_{\mathrm{y}}$ hydratisiert und oxydiert sich teilweise beim Handhaben an der Luft $\left(25^{\circ} \mathrm{C}\right)$. Unmittelbar hinter der Entladung wurde reproduzierbar die Zusammensetzung $\mathrm{CS}_{0,91} \mathrm{O}_{0,08} \cdot n \mathrm{H}_{2} \mathrm{O} \quad(n \approx 0,03)$ gefunden. Diese Präparate zersetzen sich im Hochvakuum, beginnend bei etwa $100^{\circ}$, unter Abgabe von $\mathrm{H}_{2} \mathrm{O}, \mathrm{SO}_{2}, \mathrm{CS}_{2}, \mathrm{COS}, \mathrm{CO}, \mathrm{S}_{2}$ und $\mathrm{C}_{2} \mathrm{~S} . \mathrm{S}_{2}$ konnte in $\mathrm{CS}_{2}$-Matrix isoliert werden $\left(v_{1}=674 \mathrm{~cm}^{-1}\right)$ und ist dann wesentlich stabiler als $\mathrm{CS}$. $\mathrm{C}_{2} \mathrm{~S}$ polymerisiert sofort wie oben.

Erfolglos waren Versuche, CS in heterogenen Reaktionen bei Temperaturen bis $650{ }^{\circ} \mathrm{C}$ darzustellen (Pyrolyse des $\mathrm{C}_{\mathrm{x}} \mathrm{S}_{\mathrm{y}}$, Reduktion von $\mathrm{CS}_{2}$ bzw. $\mathrm{CSCl}_{2}$ mit Metallen). Bei den bisher bekannten $\mathrm{C}_{3} \mathrm{~S}_{2}$-Darstellungsreaktionen ${ }^{5}$ entsteht das Primärprodukt CS wahrscheinlich stets in homogenen Reaktionen (thermische Dissoziation von $\mathrm{CS}_{2}$, Reduktion von $\mathrm{CS}_{2}$ mit Metalldampf). Das stets beobachtete schwarze Nebenprodukt läßt sich jetzt als $\mathrm{C}_{\mathrm{x}} \mathrm{S}_{\mathrm{y}}$ verstehen.

Aus $\mathrm{CSCl}_{2}$-Dampf läßt sich auch in der HF-Entladung kein CS darstellen, da CS in Sekundärreaktionen sofort verbraucht wird. Hauptreaktions-Produkte sind $\mathrm{CS}_{2}, \mathrm{C}_{3} \mathrm{~S}_{2}, \mathrm{CCl}_{3} \mathrm{SCl}$ und Chlorkohlenwasserstoffe, unter denen $\mathrm{C}_{2} \mathrm{Cl}_{4}$ als Hauptprodukt identifiziert wurde. $\mathrm{CCl}_{4}$ entsteht nicht. Die entscheidenden Reaktionen sind:

$$
\begin{aligned}
\mathrm{CSCl}_{2} & \rightarrow \mathrm{CS}+\mathrm{Cl}_{2} \\
3 \mathrm{CS} & \rightarrow \mathrm{C}_{3} \mathrm{~S}_{2}+\mathrm{S} \\
\mathrm{CS}+\mathrm{CSCl}_{2} & \rightarrow \mathrm{CS}_{2}+\mathrm{CCl}_{2} \\
n \mathrm{CCl}_{2} & \rightarrow \mathrm{C}_{2} \mathrm{Cl}_{4} \text { und andere } \\
\mathrm{CSCl}_{2}+\mathrm{Cl}_{2} & \rightarrow \mathrm{CCl}_{3} \mathrm{SCl} .
\end{aligned}
$$

${ }^{1}$ Die von Diallo ${ }^{2}$ für $\mathrm{C}_{3} \mathrm{~S}_{2}$ angegebenen intensiven Banden bei $860,1510,2175,2300$ und $2900-3000 \mathrm{~cm}^{-1}$ stammen von Verunreinigungen $\left(\mathrm{CS}_{2}\right.$ und wahrscheinlich $\mathrm{C}-\mathrm{H}-\mathrm{Ver}$ bindungen). Die von ihm im Zusammenhang mit dem Spektrum angestellten Erörterungen sind damit hinfällig.

2 A. O. Diallo, C. R. hebd. Séances Acad. Sci. 261, 5386 [1965].

${ }^{3}$ Zur Kopolymerisation von $\mathrm{S}_{\mathrm{n}}$ mit $\mathrm{CS}_{2}$ vergleiche 1. c. ${ }^{4}$.

${ }^{4}$ R. Steudel, Z. anorg. allg. Chem., im Druck.

5 B. v. Lengyel, Ber. dtsch. chem. Ges. 26, 2960 [1893]; A. Stock, A. Brandt u. H. Fischer, 58, 643 [1925]. 